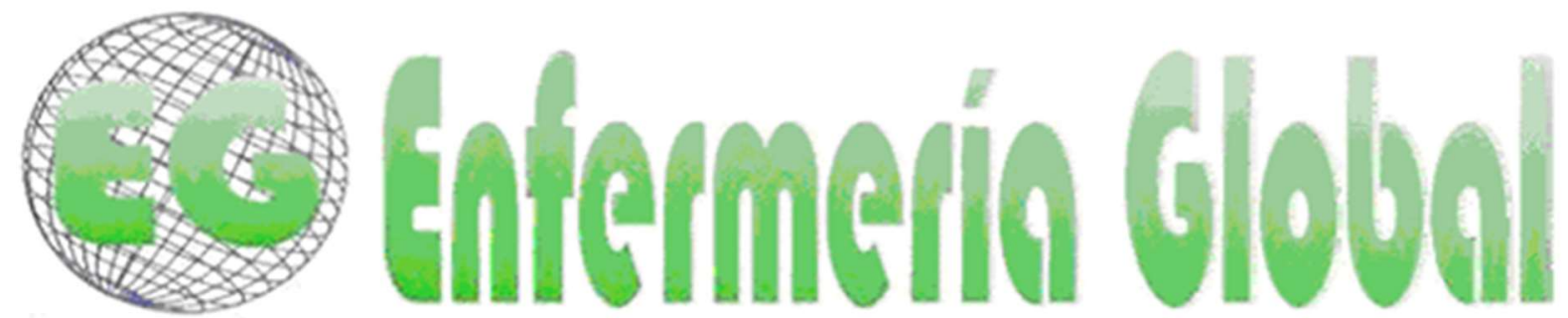

\title{
ORIGINALES
}

\section{Percepción de los pacientes sobre los cuidados de enfermería en el contexto de la crisis del COVID-19}

Patient perception of nursing care in the context of the COVID-19 crisis

\author{
Marta María Blanco-Nistal ${ }^{1}$ \\ Macrina Tortajada-Soler ${ }^{2}$ \\ Zaida Rodriguez-Puente ${ }^{3}$ \\ María Teresa Puente-Martínez ${ }^{4}$ \\ Carlos Méndez-Martínez ${ }^{5}$ \\ Jesús Antonio Fernández-Fernández ${ }^{3}$ \\ ${ }^{1}$ Centro de Salud José Aguado, León, España. \\ 2 Hospital Marina Salud de Dénia, Dénia, España. macrina-96@hotmail.com \\ ${ }^{3}$ Facultad de Ciencias de la Salud, Departamento de Enfermería y Fisioterapia Universidad de León, \\ León, España. \\ ${ }^{4}$ Enfermera de Calidad y Formación. Hospital Universitario de León, León, España. \\ ${ }^{5}$ Hospital Universitario de León, León, España.
}

\section{https://doi.org/10.6018/eglobal.479441}

\section{Recibido: $5 / 05 / 2021$}

Aceptado: $12 / 07 / 2021$

\section{RESUMEN:}

Antecedentes: La pandemia de COVID-19 ha alterado considerablemente los procedimientos habituales de los sistemas sanitarios en todo el mundo. El trabajo diario se ha vuelto física y emocionalmente agotador para los profesionales sanitarios, obligados a afrontar y adaptarse a nuevos retos y situaciones estresantes. Esta situación pesa sobre la práctica diaria de enfermería.

Objetivo: El presente estudio tuvo como objetivo determinar la percepción del paciente sobre los cuidados de enfermería humanizados recibidos durante su estancia hospitalaria.

Método: Se realizó un estudio descriptivo, cuantitativo, transversal, en el que se realizaron entrevistas telefónicas a 357 personas >18 años que ingresaron durante más de 24 horas en el Hospital de León con el fin de evaluar la percepción del paciente. Uno de los instrumentos utilizados fue la Escala de Percepción de Conductas del Cuidado de Enfermería Humanizado (PCHE), para evaluar en tres dimensiones (D): cualidades de la práctica de enfermería (D1), apertura a la comunicación enfermerapaciente (D2) y disposición al cuidado (D3). El otro instrumento utilizado fue una encuesta de satisfacción institucional para conocer la opinión sobre la calidad de la atención al cliente en el área de hospitalización.

Resultados: El porcentaje de pacientes que puntuaron como "siempre" cada dimensión fue: $\mathrm{D} 1=$ $91,2 \%$; D2 $=81,4 \%$; y D3 = 87,8\%. La satisfacción del paciente obtuvo una puntuación media de 4,6 sobre 5 .

Conclusión: A pesar del impacto negativo del COVID-19 en el sistema de salud, los pacientes percibieron la atención de enfermería humanizada recibida como muy satisfactoria. 
Palabras clave: cuidado humanizado, profesionales de enfermería, satisfacción del paciente, calidad del cuidado.

\begin{abstract}
:
Background: The COVID-19 pandemic has heavily altered regular procedures of healthcare systems worldwide. Daily work has become physically and emotionally exhausting for health care professionals, forced to face and adapt to new challenges and stressful situations. This situation weighs on the daily nursing practice and might have an impact on the quality of care provided and on the level of satisfaction perceived by hospitalized patients. Thus, the present study aimed to determine the patient's perception of humanized nursing care received during their hospital stay.

Methods: A descriptive, quantitative, cross-sectional study was carried out, in which telephone interviews were conducted in 357 people $>18$ years of age who were admitted for more than 24 hours to the Hospital de León in order to asses patient's perception. One instrument used was The Perception of Behaviors of Humanized Nursing Care Scale (PCHE), to evaluate in three dimensions (D): qualities of nursing practice (D1), openness to nurse-patient communication (D2), and willingness to care (D3). In addition, an institutional satisfaction survey was performed to know the opinion on the quality of customer service in hospitalization area.

Results: The percentage of patients scoring as "always" every dimension was: D1 $=91.2 \%$; D2= $81.4 \%$; and D3 $=87.8 \%$. Patient satisfaction obtained a mean score of 4.6 out of $5.42 .3 \%$ of population were men and $57.7 \%$ were women, most in the age range $61-75$ years. The predominant marital status and educational level were married and basic-medium, respectively.

Conclusion: Despite the negative impact of COVID-19 in the health care system, patients perceived humanized nursing care received as very successful.
\end{abstract}

Key words: humanized care; nursing staff hospital; patient satisfaction; quality of health.

\title{
INTRODUCCIÓN
}

La enfermería es una ciencia y una profesión basada en el arte de cuidar. Su pilar fundamental es el cuidado biopsicosocial y espiritual de las personas, atendiendo sus necesidades básicas en los momentos más vulnerables ${ }^{(1,2)}$. La enfermería se basa en el cuidado y la prestación de servicios de salud, con un compromiso y una responsabilidad en la prestación de los cuidados propios de esta ciencia ${ }^{(1)}$.

La teoría del cuidado humanizado, según Jean Watson, da especial importancia a la dignidad humana e incorpora conceptos como la humanización del cuidado, la asunción de valores, el cultivo de la sensibilidad y la relación de ayuda entre individuos ${ }^{(3)}$. También menciona la capacidad de los profesionales de enfermería para mostrar preocupación por las personas en todos los estados del ser, para facilitar la curación o el afrontamiento de la enfermedad, y para lograr la armonía interior. Estas capacidades se incrementan con la teoría porque orienta la práctica profesional y los resultados exitosos. Las enfermeras pueden organizar rápidamente los datos de los pacientes, decidir qué acción enfermera es la más necesaria y prestar cuidados con una expectativa de resultados ${ }^{(3)}$.

Sin embargo, como las necesidades de salud de la población han cambiado, el ámbito de la práctica enfermera, la teoría y las habilidades también deben cambiar. Las enfermeras deben asumir nuevas y complejas funciones. Lo que realmente se necesita en este mundo cambiante es garantizar un desarrollo profesional estructurado y continuo para las enfermeras que se centre en los cuidados de enfermería humanizados (4). Formalizar un modelo de humanización de los cuidados con una perspectiva global sería útil para evaluar las intervenciones de enfermería de forma coherente y mejorar los resultados ${ }^{(5)}$. 
La atención humanizada es necesaria no solo para los pacientes y sus seres queridos, sino también en el sentido contrario, para las enfermeras. Por un lado, las enfermeras de hoy deben estar altamente capacitadas en términos de educación adecuada y pensamiento crítico, con un corazón humano solidario. Pero de la misma forma, también es necesario que los pacientes y familiares aprendan a comunicar agradecimiento por el cuidado que reciben, porque esta se convierte en una forma diferente de cuidar al enfermero ${ }^{(6)}$. Los hallazgos han revelado que existen seis nobles cualidades del cuidador: amor, compromiso, empatía y simpatía, compasión, confianza y competencia, y confidencialidad y privacidad. Estas son cualidades que las enfermeras pueden usar para comunicarse en su práctica diaria con el objetivo de brindar atención humanizada efectiva a individuos, familias y grupos. Pero también tienen un efecto de vínculo de retorno sobre la percepción de pacientes y enfermeras que determina la calidad de la comunicación y la atención de enfermería ${ }^{(2)}$.

Las fallas de comunicación están asociadas con graves consecuencias para los pacientes y los equipos de salud, incluidas las enfermeras. Las enfermeras necesitan comunicarse y expresar preocupaciones profesionales y compartir un lenguaje crítico común. La comunicación eficaz puede proporcionar esperanza a los pacientes y construir relaciones profesionales adecuadas para una atención de enfermería de alta calidad ${ }^{(5)}$.

Se ha demostrado que aún existen brechas entre las expectativas de humanización por parte de las personas involucradas en el proceso de cuidado y lo que realmente sucede en la práctica clínica (5). Una comunicación de enfermería eficaz puede aumentar las esperanzas a los pacientes y establecer relaciones profesionales adecuadas para una atención de enfermería de alta calidad (5). Algunos autores sugieren que es difícil mantener los valores humanitarios en el acto asistencial, donde parecen volverse invisibles, pero las enfermeras deben esforzarse en mantener la comunicación y el apoyo a los pacientes (7). Leininger McFarland explica: "Las actitudes y la práctica del cuidado es todo lo que resalta la contribución de enfermeras de otras disciplinas" ${ }^{(8)}$.

Con el tiempo, las necesidades humanas han cambiado, al igual que los cuidados de enfermería. En las últimas décadas se ha incrementado el uso de la tecnología y la atención de enfermería se ha vuelto más específica y de mejor calidad ${ }^{(9)}$, pero este no ha sido el único factor que ha modificado la atención de enfermería.

En la práctica, los profesionales de enfermería cumplen con estándares y procedimientos, pero aún no se han desarrollado normas, o mejor, protocolos de enfermería que indiquen cómo debemos actuar en el cuidado sensible o humanizado. Actualmente, se identifica la importancia de definir líneas de acción que conduzcan a la construcción de una Política Nacional de Atención Humanizada en dos direcciones: hacia el paciente y hacia las enfermeras, para que en esta armonía se generen verdaderos ambientes sensibles a la realidad del otro ${ }^{(10)}$.

En realidad, las enfermeras se enfrentan a un nuevo desafío en el contexto de la pandemia COVID-19, ya que esta tiene efectos sobre el miedo a infectarse e infectar a otros y la dificultad de manejar las condiciones y relaciones de los pacientes en esta situación estresante. El trabajo intensivo en este contexto agota física y emocionalmente a las enfermeras, pero siguen mostrando el espíritu de dedicación y sintiendo una gran responsabilidad con los pacientes para superar la pandemia, con 
resiliencia y espíritu de compromiso profesional para superar las dificultades ${ }^{(7,8)}$. El trabajo humanizado ha tenido un impacto favorable en la salud física y psicológica, obteniendo resultados mejores en satisfacción laboral, menos enfermedades y contribuciones positivas a la salud y el bienestar (11).

Existe un instrumento validado que intenta definir estas líneas de acción y de alguna manera medir cuantitativamente el cuidado humanizado. Se determina que la Escala de Percepción de Conductas de Atención de Enfermería Humanizada (PCHE), adaptada por González-Hernández ${ }^{(12)}$, es un instrumento validado y confiable para su aplicación en el ámbito hospitalario, y está disponible para la comunidad científica a nivel nacional e internacional. Es importante que los profesionales de enfermería utilicen este tipo de instrumentos que permitan que los profesionales reflexionen sobre su disciplina y que los pacientes reciban un momento de retroalimentación sobre el cuidado, lo que contribuye al crecimiento mutuo.

A raíz de estos hallazgos, el presente trabajo plantea la hipótesis de que los pacientes del Hospital Universitario de León reciben cuidados de enfermería humanizados a pesar del contexto de crisis durante su estancia, por lo que su nivel de satisfacción también es alto. Además, la atención humanizada que perciben los pacientes ingresados en este hospital puede estar directamente relacionada con sus datos sociodemográficos y condiciones de salud, incluida la edad, el estado civil y el nivel de educación.

Debido a que el contexto de la crisis de salud es reciente, los estudios deberían mostrar si la pandemia podría afectar la atención humanizada del paciente y el nivel de satisfacción percibido. La atención de enfermería humanizada siempre ha sido una parte integral de la profesión de enfermería, y los pacientes y las enfermeras la perciben como una dinámica impulsora hacia la recuperación y la progresión profesional ${ }^{(6)}$.

Las enfermeras se enfrentan a nuevos retos en el contexto de la pandemia de COVID-19, con un trabajo intensivo y situaciones de estrés. Esto hace que las enfermeras sufran agotamiento tanto físico como psicológico y, como consecuencia, la atención y satisfacción de los pacientes pueden verse afectadas.

\section{Objetivo Principal}

- Determinar la percepción del paciente sobre los cuidados de enfermería humanizados recibidos durante su estancia en el Hospital Universitario de León.

\section{Objetivos Secundarios}

- Determinar la percepción del comportamiento de los cuidados humanizados por parte de los pacientes hospitalizados en las tres categorías diferenciadas en el instrumento utilizado.

- Identificar el nivel de satisfacción percibido por los pacientes durante el período de hospitalización.

- Establecer una relación entre la percepción del cuidado humanizado y la satisfacción con las variables sociodemográficas. 


\section{MATERIAL Y MÉTODO}

Se trata de un estudio descriptivo, cuantitativo y transversal en el que se realizaron entrevistas telefónicas con la población de estudio. Previamente se realizó una búsqueda bibliográfica de fuentes primarias y secundarias.

La población de estudio fue seleccionada aleatoriamente de 2546 pacientes, que eran todos de los pacientes ingresados en el Hospital de la Universidad de León desde el 14 de septiembre al 18 de octubre de 2020. Para llevar a cabo este estudio, hemos obtenido la autorización previa del Comité de Ética e Investigación, y posteriormente el servicio de admisión del hospital. Finalmente, se incluyeron en el estudio 357 pacientes $(n=357)$ que cumplían los siguientes criterios de inclusión y exclusión:

\section{Criterios de inclusión}

- Pacientes $\geq 18$ años ingresados en diferentes unidades hospitalarias.

- Ingresos de más de 24 horas de duración.

- Pacientes dados de alta entre el 14 de septiembre y el 18 de octubre de 2020.

- Pacientes que dieron su consentimiento oral para participar en esta investigación.

\section{Criterios de exclusión}

- Pacientes con alguna patología que cause pérdida de conciencia, daño neurológico o deterioro cognitivo.

- Pacientes ingresados en unidades psiquiátricas.

- Pacientes con impedimentos del habla.

- Pacientes fallecidos durante el tiempo transcurrido entre el alta y la entrevista telefónica.

La muestra obtenida se estimó probabilísticamente con un intervalo de confianza del $95 \%$ y un error del $5 \%$.

Las encuestas se realizaron en los meses de diciembre de 2020 y enero de 2021.

\section{Técnica y herramientas de recopilación de información}

Para la recolección de datos se utilizaron 3 instrumentos. Primero, se utilizó un cuestionario con datos sociodemográficos y clínicos, junto con una escala denominada Percepción de Conductas de Atención de Enfermería Humanizada (PCHE), 3a versión, validada por análisis factorial exploratorio por González en $2015^{(12)}$ y una encuesta de satisfacción institucional, Opinión del Área de Hospitalización de Calidad de Atención al Cliente de CAULE.

La tercera versión del PCHE consiste en un cuestionario de 32 ítems con una escala Likert de 4 puntos con valores asignados a las preguntas de la siguiente manera: nunca $=1$ punto, a veces $=2$ puntos, casi siempre $=3$ puntos y siempre $=4$ puntos. Tiene una puntuación máxima de 168 puntos y una mínima de 42.

Además, la $3 a$ versión tiene validez de contenido, teniendo un índice de validez de contenido de 0.98 por expertos, y validez de constructo a través de la medición de un análisis factorial exploratorio que generó tres categorías o dimensiones denominadas: 
cualidades del hacer de enfermería, apertura a la comunicación enfermera-paciente y disposición para la atención, que miden el constructo del instrumento, Conductas de cuidado humanizado. Finalmente, se determinó que el instrumento PCHE a su nueva versión, es confiable, ya que se obtuvo un alfa de Cronbach de $0.96{ }^{(12)}$

La encuesta de satisfacción institucional (Anexo B) evalúa las opiniones sobre la calidad de la atención al cliente a través de 11 ítems con una escala Likert de 5 puntos, puntuando desde $1=$ muy mala hasta $5=$ muy buena. También se incluyó el nivel general de satisfacción (ítem 12), con una escala Likert de 4 puntos: $1=$ nada satisfecho, 2 = un poco satisfecho, 3 = satisfecho y 4 = muy satisfecho.

También se recopilaron datos sociodemográficos, incluyendo edad, sexo, estado civil y nivel educativo.

La técnica utilizada fueron las llamadas telefónicas entre noviembre y diciembre de 2020 , en las que se definió el carácter voluntario y anónimo de la participación y se solicitó explícitamente el consentimiento de los participantes. Los autores del estudio recopilaron datos de los participantes que cumplieron con los criterios de inclusión y completaron todos los ítems por teléfono con consentimiento informado previo. Posteriormente, los participantes fueron codificados con números, para respetar el anonimato y la confidencialidad de los datos.

\section{Procesamiento y análisis de datos}

Se elaboró una matriz de datos en Microsoft Excel y posteriormente se analizaron los datos con el programa Epi Info ${ }^{\text {TM }}$ y SPSS Statistics 26.0. Los resultados se presentaron en tablas estadísticas para su análisis. Se utilizó estadística descriptiva en forma de tablas de frecuencia, medidas de tendencia central, tablas de contingencia y análisis de significancia.

\section{Consideraciones éticas}

Este proyecto fue aprobado por el Comité de Ética e Investigación y respetó los principios éticos sustentados en el Informe Belmont y la Declaración de Helsinki, garantizándose el derecho a la confidencialidad. Se obtuvo el consentimiento informado de todos los sujetos involucrados en el estudio. Los autores declaran no tener conflicto de intereses.

\section{RESULTADOS}

\section{Variables sociodemográficas}

El perfil de los usuarios encuestados $(n=357)$ se caracteriza por una media de edad de 61,3 años, con una mediana de 64,2 años, un máximo de 93,6 años y un mínimo de 18,2 años. Las edades se distribuyen en cuatro rangos (Tabla 1), donde el rango más representado es el de 61 a 75 años (31\%) seguido del rango de 46 a 60 años (25\%), de $>75$ años (21\%), de 31 a 45 años (19\%), y el menos representado de 18 a 30 años (4\%). 
Gráfico 1. Distribución por rango de edad.

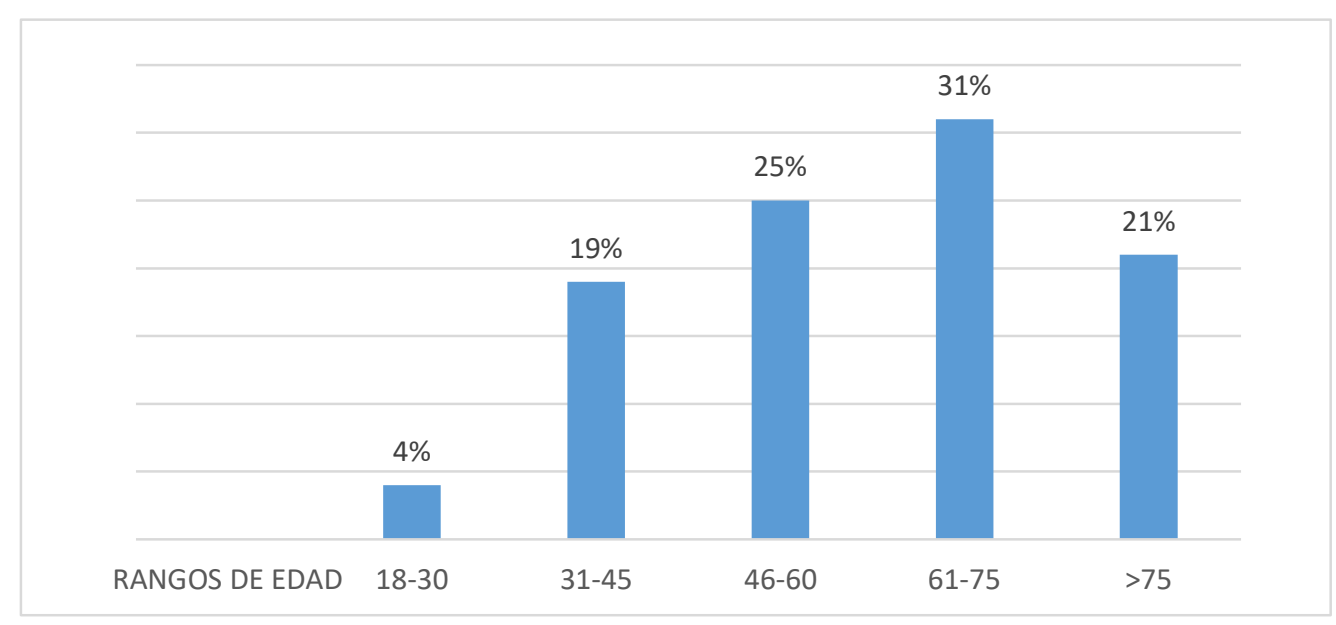

Además, el $42,3 \% \%$ son hombres y el $57,7 \%$ son mujeres cuya distribución, según su estado civil, se recoge en la Tabla 1; destaca el estado civil casado por encima de los demás, con datos que superan la mitad de los encuestados $(58,82 \%)$, y con resultados homogéneos entre sexos. El caso de los viudos es el contrario, están escasamente representados (15,97\%), existiendo importantes diferencias entre géneros, el $14,04 \%$ de los viudos son hombres y el $85,96 \%$ son mujeres.

Tabla 1. Distribución por género y estado civil.

\begin{tabular}{c|ccc}
\hline Estado civil & Hombres & Mujeres & Total \\
\hline Solterola & 23 & 37 & 60 \\
$\%$ Específico & $38.33 \%$ & $61.67 \%$ & $100.00 \%$ \\
$\%$ Global & $15.23 \%$ & $17.96 \%$ & $16.81 \%$ \\
\hline Divorciado/a & 12 & 18 & 30 \\
\% Específico & $40.00 \%$ & $60.00 \%$ & $100.00 \%$ \\
\hline Casado/a & $7.95 \%$ & $8.74 \%$ & $8.40 \%$ \\
\hline$\%$ Específico & 108 & 102 & 210 \\
\hline$\%$ Global & $71.52 \%$ & $48.57 \%$ & $100.00 \%$ \\
\hline Viudo/a & 8 & $49.51 \%$ & $58.82 \%$ \\
\hline$\%$ Específico & $14.04 \%$ & 49 & 57 \\
\hline$\%$ Global & $5.30 \%$ & $23.79 \%$ & $100.00 \%$ \\
\hline
\end{tabular}




\begin{tabular}{l|ccc}
\hline \multicolumn{1}{l|}{ Total } & 151 & 206 & 357 \\
\% Específico & $42.30 \%$ & $57.70 \%$ & $100.00 \%$ \\
\% Global & $100.00 \%$ & $100.00 \%$ & $100.00 \%$ \\
\hline
\end{tabular}

Respecto a las frecuencias de nivel de estudios por género (Tabla 2.), casi el $80 \%$ de los encuestados tienen un nivel básico-medio de educación (47,90\% básico y $30,53 \%$ medio), mientras que solamente el $20,45 \%$ posee un nivel académico o universitario, y el $4 \%$ máster/ doctorado. Presentan una distribución por géneros bastante homogénea en los niveles básico y medio, sin embargo, en el nivel académico universitario, casi el $65 \%$ de la muestra son mujeres.

Tabla 2. Distribución por género y nivel de estudios.

\begin{tabular}{r|ccc}
\hline \multicolumn{1}{c|}{$\begin{array}{c}\text { Nivel de } \\
\text { educación }\end{array}$} & Hombres & Mujeres & Total \\
\hline Básico & 72 & 99 & 171 \\
$\%$ Específico & $42.11 \%$ & $57.89 \%$ & $100.00 \%$ \\
$\%$ Global & $47.68 \%$ & $48.06 \%$ & $47.90 \%$ \\
\hline Medio & 53 & 56 & 109 \\
\% Específico & $48.62 \%$ & $51.38 \%$ & $100.00 \%$ \\
\% Global & $35.10 \%$ & $27.18 \%$ & $30.53 \%$ \\
\hline Universitario & 26 & 47 & 73 \\
\% Global & $17.22 \%$ & $22.82 \%$ & $20.45 \%$ \\
\hline Máster/Doctorado & 0 & 4 & 4 \\
\% Específico & $0.00 \%$ & $100.00 \%$ & $100.00 \%$ \\
\% Global & $0.00 \%$ & $1.94 \%$ & $1.12 \%$ \\
\hline Total & 151 & 206 & 357 \\
\hline & $42.30 \%$ & $57.70 \%$ & $100.00 \%$ \\
\hline
\end{tabular}




\section{Percepción de los pacientes de la humanización en los cuidados de las enfermeras/os}

Se evaluaron los comportamientos de cuidado humanizado de las enfermeras/os en sus tres dimensiones y en la percepción global. En la Escala PCHE los pacientes eligieron entre cuatro opciones (nunca, algunas veces, casi siempre, siempre). En la Tabla 3 se reflejan los porcentajes de respuestas por ítems y los promedios de la dimensión 1 (D1), definida como "cualidades del hacer de enfermería". En la D1 se destacan aspectos como facilitar que el paciente experimente sensaciones de bienestar y confianza, logrando que el paciente perciba un vínculo respetuoso en la relación enfermera paciente. Un promedio del $91 \%$ de los pacientes encuestados "siempre" han percibido los cuidados referentes a esta dimensión. El ítem peor puntuado fue el P15 "le explican los cuidados usando un tono de voz adecuado", donde un $88,0 \%$ de los pacientes respondió que "siempre".

Tabla 3. Dimensión 1. Cualidades del hacer de enfermería.

\begin{tabular}{c|cc|cc|cc|cc}
\hline & \multicolumn{2}{|c|}{ Siempre } & \multicolumn{2}{c|}{ Casi siempre } & \multicolumn{2}{c|}{ Algunas veces } & \multicolumn{2}{c}{ Nunca } \\
\hline & $\mathrm{n}$ & $\%$ & $\mathrm{n}$ & $\%$ & $\mathrm{n}$ & $\%$ & $\mathrm{n}$ & $\%$ \\
\hline P1 & 332 & $93.0 \%$ & 18 & $5.0 \%$ & 5 & $1.4 \%$ & 2 & $0.6 \%$ \\
P2 & 325 & $91.0 \%$ & 22 & $6.2 \%$ & 10 & $2.8 \%$ & 0 & $0.0 \%$ \\
P6 & 330 & $92.4 \%$ & 18 & $5.0 \%$ & 8 & $2.2 \%$ & 1 & $0.3 \%$ \\
P7 & 325 & $91.0 \%$ & 21 & $5.9 \%$ & 9 & $2.5 \%$ & 2 & $0.6 \%$ \\
P8 & 322 & $90.2 \%$ & 22 & $6.2 \%$ & 10 & $2.8 \%$ & 3 & $0.8 \%$ \\
P15 & 314 & $88.0 \%$ & 29 & $8.1 \%$ & 11 & $3.1 \%$ & 3 & $0.8 \%$ \\
P17 & 330 & $92.4 \%$ & 17 & $4.8 \%$ & 7 & $2.0 \%$ & 4 & $1.1 \%$ \\
\hline Total & 325 & $91.2 \%$ & 21 & $5.9 \%$ & 9 & $2.4 \%$ & 2 & $0.6 \%$ \\
\hline
\end{tabular}

En la Tabla 4 se reflejan los porcentajes de respuestas por ítem y los promedios de la dimensión 2 (D2), definida como "apertura a la comunicación enfermera/o-paciente". Esta dimensión se refiere a un proceso dinámico, fundamental para el crecimiento, el cambio y la conducta, que permiten la interacción con el sujeto de cuidado a través de habilidades comunicativas que facilitan la transmisión de una realidad y la interacción con la misma. Esta apertura se orienta a la escucha activa, al diálogo, a la presencia y la compresión de quien es cuidado. En esta dimensión está el ítem menos valorado de la Escala (P12) "le indican su nombre y cargo antes de realizar los procedimientos", donde el 59,7\% refiere "siempre", el 14\% "casi siempre", el 16,8\% refiere "a veces" y el 9\% "nunca". En el resto de ítems entre el $79 \%$ y el $87,4 \%$ de los encuestados percibido los cuidados humanitarios referentes a esta dimensión "siempre", con un promedio del $81,4 \%$. 
Tabla 4. Dimensión 2. Apertura a la comunicación enfermera/o-paciente.

\begin{tabular}{c|cc|cc|cc|cc}
\hline & \multicolumn{2}{|c|}{ Siempre } & \multicolumn{2}{c|}{ Casi siempre } & \multicolumn{2}{c}{ Algunas veces } & \multicolumn{2}{c}{ Nunca } \\
\hline & $\mathrm{n}$ & $\%$ & $\mathrm{n}$ & $\%$ & $\mathrm{n}$ & $\%$ & $\mathrm{n}$ & $\%$ \\
\hline P4 & 282 & $79.0 \%$ & 55 & $15.4 \%$ & 16 & $4.5 \%$ & 2 & $0.6 \%$ \\
P5 & 300 & $84.0 \%$ & 37 & $10.4 \%$ & 14 & $3.9 \%$ & 6 & $1.7 \%$ \\
P10 & 308 & $86.3 \%$ & 32 & $9.0 \%$ & 12 & $3.4 \%$ & 5 & $1.4 \%$ \\
P11 & 301 & $84.3 \%$ & 36 & $10.1 \%$ & 13 & $3.6 \%$ & 7 & $2.0 \%$ \\
P12 & 213 & $59.7 \%$ & 50 & $14.0 \%$ & 60 & $16.8 \%$ & 32 & $9.0 \%$ \\
P14 & 305 & $85.4 \%$ & 33 & $9.2 \%$ & 15 & $4.2 \%$ & 4 & $1,1 \%$ \\
P19 & 304 & $85.2 \%$ & 30 & $8.4 \%$ & 15 & $4.2 \%$ & 8 & $2.2 \%$ \\
\hline Total & 291 & $81.4 \%$ & 37 & $10.5 \%$ & 20 & $5.7 \%$ & 8 & $2.3 \%$ \\
\hline
\end{tabular}

En la Tabla 5 se reflejan los porcentajes de respuestas por ítem de la dimensión 3 (D3), definida como "disposición para la atención". Esta dimensión se refiere a la disposición que surge de ser solicitado por el paciente, que no se limita a un acto de observar, sino que requiere una inmersión en su realidad para descubrir sus necesidades y fortalecer el vínculo que los une en el cuidado. Un promedio del $87,8 \%$ de los encuestados ha contestado que percibe los cuidados "siempre". En esta dimensión está el ítem más valorado (P30) "le indican que cuando quiera algo, usted puede llamar" en el que el 99,4\% de los pacientes han indicado "siempre".

Tabla 5. Dimensión 3: Disposición para la atención.

\begin{tabular}{c|cc|cc|cc|cc}
\hline & \multicolumn{2}{|c|}{ Siempre } & \multicolumn{2}{c|}{ Casi siempre } & \multicolumn{2}{c|}{ Algunas veces } & \multicolumn{2}{c}{ Nunca } \\
\hline & $\mathrm{n}$ & $\%$ & $\mathrm{n}$ & $\%$ & $\mathrm{n}$ & $\%$ & $\mathrm{n}$ & $\%$ \\
\hline P3 & 311 & $87.1 \%$ & 23 & $6.4 \%$ & 15 & $4.2 \%$ & 6 & $1.7 \%$ \\
P13 & 298 & $83.5 \%$ & 33 & $9.2 \%$ & 21 & $5.9 \%$ & 5 & $1.4 \%$ \\
P16 & 317 & $88.8 \%$ & 31 & $8.7 \%$ & 9 & $2.5 \%$ & 4 & $1.1 \%$ \\
P18 & 330 & $92.4 \%$ & 20 & $5.6 \%$ & 9 & $2.5 \%$ & 2 & $0.6 \%$
\end{tabular}




\begin{tabular}{|c|c|c|c|c|c|c|c|c|}
\hline P20 & 301 & $84.3 \%$ & 7 & $2.0 \%$ & 4 & $1.1 \%$ & 0 & $0.0 \%$ \\
\hline P21 & 290 & $81.2 \%$ & 43 & $12.0 \%$ & 11 & $3.1 \%$ & 9 & $2.5 \%$ \\
\hline P22 & 298 & $83.5 \%$ & 37 & $10.4 \%$ & 19 & $5.3 \%$ & 6 & $1.7 \%$ \\
\hline P23 & 387 & $80.4 \%$ & 38 & $10.6 \%$ & 13 & $3.6 \%$ & 17 & $4.8 \%$ \\
\hline P24 & 308 & $86.3 \%$ & 29 & $8.1 \%$ & 13 & $3.6 \%$ & 4 & $1.1 \%$ \\
\hline P25 & 288 & $80.7 \%$ & 27 & $7.6 \%$ & 21 & $5.9 \%$ & 20 & $5.6 \%$ \\
\hline P26 & 310 & $86.8 \%$ & 29 & $8.1 \%$ & 15 & $4.2 \%$ & 3 & $0.8 \%$ \\
\hline P27 & 318 & $89.1 \%$ & 24 & $6.7 \%$ & 11 & $3.1 \%$ & 3 & $0.8 \%$ \\
\hline P28 & 322 & $90.2 \%$ & 24 & $6.7 \%$ & 10 & $2.8 \%$ & 1 & $0.3 \%$ \\
\hline P29 & 321 & $89.9 \%$ & 20 & $5.6 \%$ & 8 & $2.2 \%$ & 8 & $2.2 \%$ \\
\hline P30 & 355 & $99.4 \%$ & 16 & $4.5 \%$ & 5 & $1.4 \%$ & 1 & $0.3 \%$ \\
\hline P31 & 331 & $92.7 \%$ & 16 & $4.5 \%$ & 9 & $2.5 \%$ & 1 & $0.3 \%$ \\
\hline P32 & 341 & $95.5 \%$ & 9 & $2.5 \%$ & 7 & $2.0 \%$ & 0 & $0.0 \%$ \\
\hline Total & 313 & $87.8 \%$ & 25 & $7.0 \%$ & 12 & $3.3 \%$ & 5 & $1.5 \%$ \\
\hline
\end{tabular}

En la percepción global de los pacientes (Tabla 6), se evalúan los comportamientos de cuidado humanizado en sus tres dimensiones. Resultando que en un $86,9 \%$ de los casos, los pacientes "siempre" perciben unos cuidados humanizados, en un 7,6\% "casi siempre", en un 3,7\% "algunas veces" y "nunca" en un 1,5\%.

Tabla 6. Distribución general de la percepción de los pacientes de la humanización de los cuidados de las enfermeras/os.

\begin{tabular}{|c|c|c|c|c|c|c|c|}
\hline \multicolumn{2}{|c|}{ Siempre } & \multicolumn{2}{|c|}{ Casi siempre } & \multicolumn{2}{|c|}{ Algunas veces } & \multicolumn{2}{|c|}{ Nunca } \\
\hline $\mathrm{n}$ & $\%$ & $\mathrm{~N}$ & $\%$ & $\mathrm{~N}$ & $\%$ & $\mathrm{~N}$ & $\%$ \\
\hline 310 & $86.9 \%$ & 27 & $7.6 \%$ & 13 & $3.7 \%$ & 5 & $1.5 \%$ \\
\hline
\end{tabular}

Otra forma de mostrar la percepción del cuidado humanizado de las enfermeras, es valorando la puntuación en tres rangos (buena, regular, mala). En este formato, más del $90 \%$ de los pacientes hospitalizados muestran una percepción buena en todas las dimensiones. La dimensión 2 de "apertura a la comunicación enfermera/o - paciente" ha sido la peor puntuada con un $91,9 \%$ y la más puntuada con un $96,6 \%$ ha sido la dimensión 1 "cualidades del hacer de enfermería" (Tabla 7). 
Tabla 7. Valoración global de la percepción de los pacientes de la humanización de los cuidados de enfermería por dimensión.

\begin{tabular}{|c|c|c|c|c|c|}
\hline & Dimensión & Valoración & Rangos & $\mathbf{n}$ & $\%$ \\
\hline \multirow[t]{3}{*}{ D1 } & Cualidades del & Buena & $21-28$ & 345 & $96.6 \%$ \\
\hline & enfermería & Moderada & $14-20$ & 11 & $3.1 \%$ \\
\hline & & Mala & $7-13$ & 1 & $0.3 \%$ \\
\hline \multirow[t]{3}{*}{ D2 } & Apertura a la & Buena & $24-32$ & 328 & $91.9 \%$ \\
\hline & $\begin{array}{l}\text { comunicación } \\
\text { enfermera/o - }\end{array}$ & Moderada & $16-23$ & 24 & $6.7 \%$ \\
\hline & paciente & Mala & $8-15$ & 5 & $1.4 \%$ \\
\hline \multirow[t]{6}{*}{ D3 } & Disposición para & Buena & $51-68$ & 337 & $94.4 \%$ \\
\hline & & Moderada & $34-50$ & 18 & $5.0 \%$ \\
\hline & & Mala & $17-33$ & 2 & $0.6 \%$ \\
\hline & Percepción & Buena & $96-128$ & 336 & $94.1 \%$ \\
\hline & global & Moderada & $64-95$ & 20 & $5.6 \%$ \\
\hline & & Mala & $32-63$ & 1 & $0.3 \%$ \\
\hline
\end{tabular}

\section{Nivel de satisfacción de los usuarios}

La importancia de conocer la perspectiva del paciente respecto a los servicios ha sido reconocida durante la década de los 80 y ha ido incrementándose ${ }^{(38)}$. La satisfacción se asocia comúnmente con la calidad, que constituye una amplia exigencia, tradicionalmente centrada en la institución y actualmente extendida a la perspectiva de los usuarios y trabajadores ${ }^{(40)}$. Está formada por tres elementos: rendimiento percibido, expectativas del usuario y los niveles de satisfacción.

El instrumento utilizado en el presente trabajo es institucional, y evalúa estos tres elementos distribuidos en 11 ítems (Gráfico 2) evaluados mediante escala Likert (1-5). El ítem 2 es el menos puntuado con un promedio de 4,1 (PSAT2) correspondiente a "le informaron explícitamente sobre sus derechos" y el más puntuado es el ítem 10 con un promedio de 4,8 (PSAT10). Destaca que los resultados totales en satisfacción muestran un nivel muy alto con un promedio de 4,6 sobre 5, acorde con el ítem 12 "percepción de nivel de satisfacción" que tiene un promedio de 3,8 sobre 4. 
Gráfico 2. Nivel de satisfacción por ítem y total promedio.

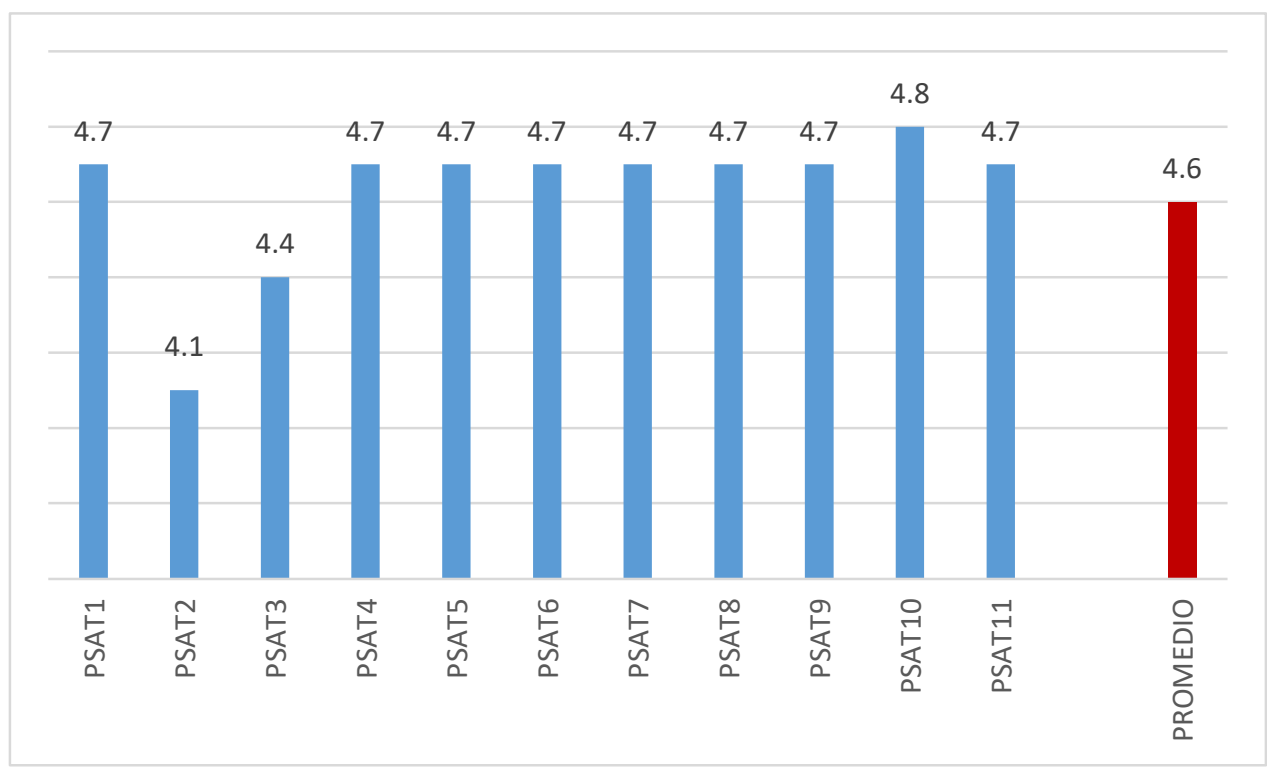

En el cruce de resultados de la satisfacción con el género $(42,3 \%$ hombres vs. $57,7 \%$ mujeres) no se aprecian diferencias significativas; tampoco se aprecian diferencias entre satisfacción y rango de edad. Los mejores resultados (5 puntos) varían entre el $72,7 \%$ del rango de edad $>75$ años, frente al $80 \%$ del rango $31-45$ años, el rango que mejor ha puntuado la satisfacción. Los peores resultados (1 punto) también corresponde al rango de edad $>75$ años, aunque solo el 1,3\% califica la satisfacción con un promedio tan bajo. En cuanto a la satisfacción por estado civil no son resultados representativos, ya que no existe homogeneidad entre el estado civil casado y el resto. 
Gráfico 3. Distribución de la satisfacción por rango de edad.

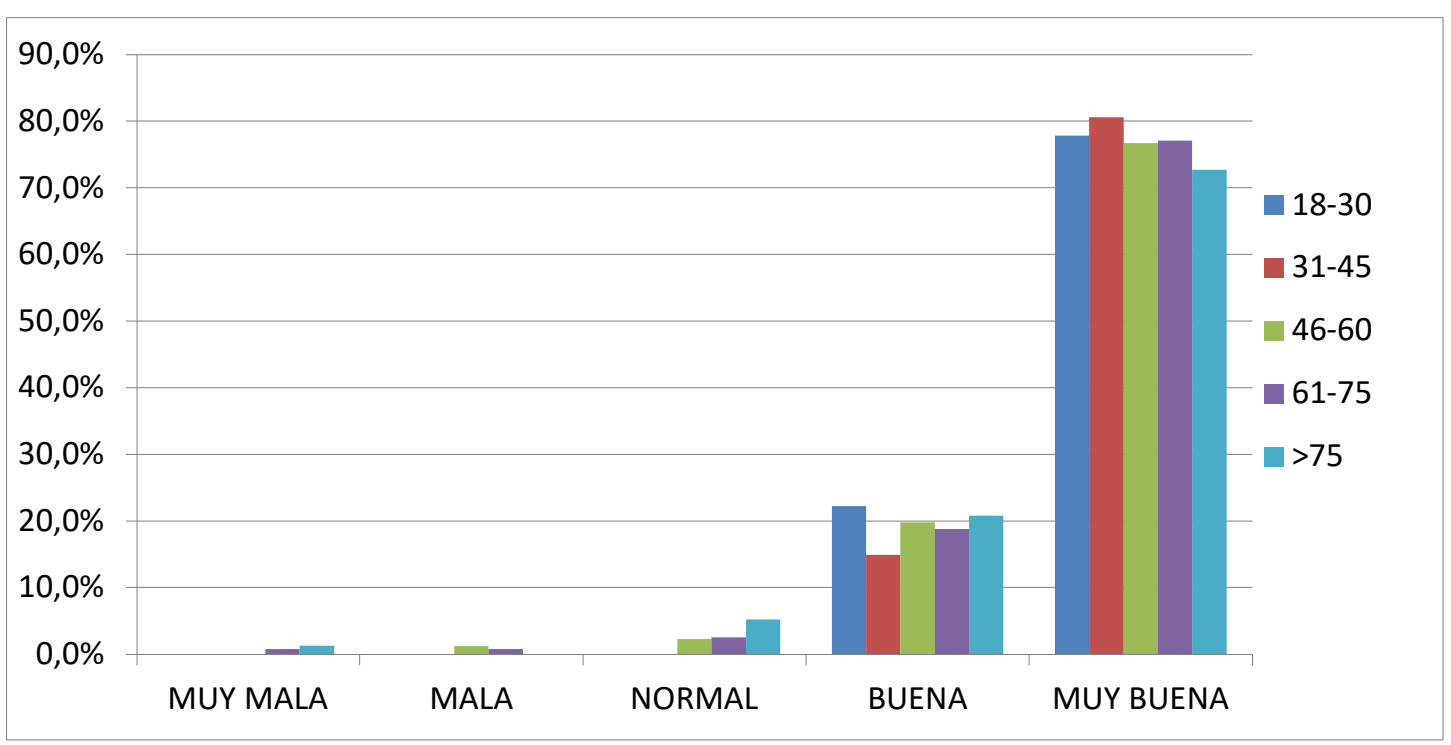

\section{DISCUSIÓN}

El objetivo de este estudio fue determinar la percepción de los pacientes hospitalizados sobre los cuidados humanizados recibidos por las enfermeras, identificar sus variables sociodemográficas y conocer su nivel de satisfacción con los cuidados recibidos.

En primer lugar, la población de estudio fue una muestra homogénea en cuanto a género, a pesar de tener más mujeres que hombres. Estos resultados coinciden con los de autores como Monje et al. (1), Eugenio et al. (15), Romero-Massa et al. (16) y Valdivia ${ }^{(17)}$. Como defienden estos autores, estos datos están en consonancia con la población mundial, donde encontramos un mayor porcentaje de mujeres que de hombres.

Por lo que se refiere al estado civil, casado es el estado civil dominante con respecto al resto, y resulta ser un grupo muy homogéneo entre ambos géneros. Disconformidad existe en el estado civil "viudo" cuyo mayor porcentaje lo obtiene el sexo mujer con un $85,96 \%$ respecto a los hombres. Esto puede deberse a la mayor esperanza de vida de las mujeres frente a los hombres. Cabe destacar que el grupo minoritario fue el de divorciado, coincidiendo así con un estudio anterior de Valdivia ${ }^{(17)}$.

En nuestro estudio, el nivel de estudios básico-medio tiene una incidencia mayor que para el resto, en contraposición con los datos obtenidos para los niveles de estudios académico/universitario y máster/doctorado, que tienen una incidencia menor. Eugenio et al. (15) obtuvieron resultados diferentes a los nuestros, ya que su estudio mostró niveles de educación técnica y universitaria más altos que para los de básicos y medios.

La $3^{a}$ versión de la Escala de Percepción de Comportamientos de Cuidados Humanizados de Enfermería (PCHE) divide los cuidados humanizados 
proporcionados por el personal de enfermería en tres dimensiones: cualidades de la práctica de enfermería (D1), apertura a la comunicación enfermera-paciente (D2) y disposición al cuidado (D3).

En el presente estudio, D1 ha sido la mejor valorada, mientras que la que ha obtenido una puntuación más baja ha sido D2. Estos datos son similares a los obtenidos en otros estudios ${ }^{(16,17)}$ en los que D1 tuvo el valor más alto, pero en su estudio la dimensión con una puntuación menor resulta ser la D3, difiriendo así del nuestro. En cuanto a la percepción global de la atención, más del $85 \%$ de los pacientes respondieron "siempre", lo que también coincide con otros resultados ${ }^{(18,19)}$, aunque el porcentaje es ligeramente superior en el presente estudio ${ }^{(6,8,9)}$.

Además, el instrumento PCHE evalúa de manera indirecta los derechos y deberes que tienen las personas en relación con acciones vinculadas a su salud: derecho a un trato digno (ítem 4, 5, 12 y 24); derecho a la compañía, asistencia espiritual y a la atención con pertinencia cultural (ítems 17 y 23); derecho a la autonomía (ítems 19 y 29). Estos derechos y deberes están reflejados en la Ley 41/2002, de 14 de noviembre, básica reguladora de la autonomía del paciente y de derechos y obligaciones en materia de información y documentación clínica, a nivel nacional; y la Ley $8 / 2003$, de 8 de abril, sobre derechos y deberes de las personas en relación con la salud, a nivel autonómico. Todos los ítems mencionados fueron calificados mayoritariamente con un "siempre" en nuestros resultados obtenidos.

Por otro lado, también medimos el nivel de satisfacción de los pacientes mediante una escala de Likert. En este estudio, obtuvimos una puntuación media de 4,6 sobre 5, siendo la puntuación más alta para el ítem 10 de 4,8 sobre 5 y la más baja para el ítem 2 de 4,1 sobre 5. La puntuación promedia, referida al ítem 12, fue de 3,8 sobre 4. Los resultados muestran un mayor grado de satisfacción de los pacientes frente a estudios similares que han mostrado puntuaciones significativamente más bajas en satisfacción (16), y son muy similares entre los rangos de edad estudiados.

Creemos que algunos ítems, como el 2 y el 3, podrían mejorarse ya que están por debajo de la media de satisfacción de nuestro estudio. En general, el hallazgo destacable del presente trabajo es que, a pesar de la crisis sanitaria a la que estaban expuestos los pacientes, los resultados obtenidos para los cuidados humanizados de enfermería y la satisfacción de los pacientes son muy satisfactorios según la hipótesis propuesta en el estudio. Un estudio sobre los cuidados humanizados indicó que esta percepción también depende de las circunstancias de la modalidad y del momento y lugar de los hechos (17). Así, es posible que los pacientes que han vivido una crisis sanitaria hayan considerado el contexto de la pandemia.

Existe controversia en la percepción contraria, el comportamiento humanizado que las enfermeras creen que pueden aportar. A pesar de que los estudios apoyan una gran resiliencia en la enfermería y un espíritu de dedicación profesional para superar las dificultades, se ha demostrado una baja percepción de los cuidados humanizados por parte de las propias enfermeras ${ }^{(18)}$. Por lo tanto, existen discrepancias curiosas en cuanto a la percepción de los cuidados humanizados entre las enfermeras o los cuidadores y los pacientes que reciben dichos cuidados, como demostró un estudio con la herramienta PCHE (19). Frente a la idea de Leininger, citada por MacFarland, de que "la atención culturalmente congruente es la que deja al paciente convencido de que recibe una buena atención"(20), la atención humanizada significa algo más que 
recibir un buen trato o tener satisfacción; significa también que "el otro" debe cuidarse para que el equipo de enfermería y el paciente obtengan grandes resultados. Es lo que perciben el paciente y la familia, así como el profesional y el equipo que presta los cuidados"(8).

También debemos tener en cuenta que las enfermeras se enfrentan a nuevos retos en el contexto de la pandemia de COVID-19, con efectos derivados del trabajo intenso que agotan a las enfermeras física y emocionalmente. Pensamos que una futura línea de investigación podría ser estudiar las percepciones de las conductas humanizadas por parte de las enfermeras de nuestro hospital, e investigar de nuevo ambos tipos de percepciones fuera del contexto de la crisis sanitaria, para poder compararlas dentro y fuera del contexto pandémico.

Además, proponemos desarrollar estrategias de cuidados humanizados entre los profesionales, que deben ser liderados por las enfermeras y realizados con un enfoque biopsicosocial para evitar la tecnificación de los cuidados. Del mismo modo, también es necesario conocer el nivel de formación universitaria que reciben los futuros profesionales de enfermería para resaltar su importancia.

\section{Limitaciones}

Aunque el presente manuscrito aporta una valiosa información sobre el grado de satisfacción del paciente respecto a los cuidados de enfermería recibidos durante su estancia en el hospital, el estudio tiene ciertas limitaciones. Debido a la situación de pandemia, tuvimos que realizar entrevistas telefónicas en lugar de conversaciones cara a cara, y no sabemos si eso podría haber influido en las respuestas.

Además, la población de la muestra tenía límites geográficos, por lo que los resultados y las conclusiones de nuestro estudio no pueden extrapolarse de forma general.

\section{CONCLUSIÓN}

Se ha obtenido una muestra homogénea, con algo más de mujeres que hombres y más representación en el rango de edad de 61 a 75 años. En la población del estudio, el estado civil predominante ha sido casado y el minoritario, divorciado. El nivel de educación ha sido principalmente básico-medio con un porcentaje de casi el $80 \%$.

La percepción del cuidado humanizado fue evaluada por la $3^{\text {a }}$ versión del PCHE, cuyos ítems se dividen en tres categorías o dimensiones: cualidades del hacer de enfermería (D1), apertura a la comunicación enfermera-paciente (D2) y disposición para la atención (D3). Para D1, el 91,2\% de los encuestados eligió "siempre", el $81,4 \%$ para D2 y el $87,8 \%$ para D3. El valor medio de la percepción general de "siempre" fue del 86,9\%.

La satisfacción del paciente se evaluó con una encuesta institucional, obteniendo una puntuación media de 4,6 sobre 5 . La puntuación más baja fue 4,1 en el ítem 2 y la más alta fue de 4,8 en el ítem 10. El nivel percibido de satisfacción global obtuvo una puntuación de 3,8 sobre 4 en el ítem 12 . 
Con los resultados obtenidos en el presente trabajo identificamos las fortalezas y debilidades de las variables estudiadas, pero el hallazgo destacable es que a pesar de la crisis de salud a la que estuvieron expuestos los pacientes, los resultados en la atención de enfermería humanizada y la satisfacción del paciente son muy exitosos, siguiendo la línea de la hipótesis planteada en el estudio. Es posible que los pacientes consideren el contexto pandémico al responder, pero sin duda los resultados muestran de manera indirecta que las enfermeras continúan desarrollando un espíritu de dedicación y resiliencia para superar las dificultades con el propósito de promover el bienestar del paciente.

La literatura revisada muestra que, en cuanto a la calidad de la atención y las conductas humanizadas, es tan importante la percepción de los pacientes y sus familias como la percepción que tienen los profesionales de la asistencia que brindan. Este hallazgo es la base de una línea de investigación futura muy importante para nosotros, donde se puede aplicar el mismo instrumento PCHE para conocer la percepción de cuidados humanizados que piensan que dan los profesionales.

La campaña Nursing Now ha mostrado al mundo el valor de la atención de enfermería durante la pandemia de COVID-19. Queremos contribuir también con nuestra investigación, y aportar datos reales que justifiquen el gran papel de las enfermeras en la sociedad.

\section{REFERENCIAS}

1. Monje, P.; Miranda, P.; Oyarzún, J:; Fredy Seguel, P. \& Flores, E. Perception of humanized nursing care by hospitalized users. Ciencia y Enfermeria. 2018;24:1-10.

2. Kheokao, J.; Krirkgulthorn, T.; Umereweneza, S. \& Seetangkham, S. Communication Factors in Holistic Humanized Nursing Care: Evidenced from Integrative Review. Journal of MCU Peace Studies. 2019;7(3):609-27.

3. Urra, M.E.; Jana, A. \& García,V. Algunos Aspectos Esenciales Del Pensamiento De Jean Watson Y Su Teoría De Cuidados Transpersonales. Ciencia y enfermería. 2011;17(3):11-22.

4. Marriner Tomey, A. \& Raile Alligood, M. Modelos y teorías en enfermería. 6a. Elsevier, editor. España; 2014.

5. Button, D.; Harrington, A. \& Belan, I. E-learning \& information communication technology (ICT) in nursing education: A review of the literature. Nurse Education Today. 2014;34(10):1311-23.

6. Hung, H.Y; Huang Y.F.; Tsai, J.J. \& Chang, Y.J. Current state of evidencebased practice education for undergraduate nursing students in Taiwan: A questionnaire study. Nurse Education Today. 2015;35(12):1262-7.

7. Liu, Q.; Luo, D.; Haase, J.E:; Guo, Q.; Wang, X.; Liu, S.; Xia, L.; Liu, Z.; Yan, J. The experiences of health-care providers during the COVID-19 crisis in China: a qualitative study. Lancet Glob Health. 2020;8:e790-98.

8. McFarland M. Teoría de la diversidad y de la universalidad de los cuidados culturales. Modelos y teorías en enfermería 6a ed. Madrid. 2007. 472-498 p.

9. Busch, I.M.; Moretti, F.; Travaini, G.; Wu, A.W. \& Rimondini, M. Humanization of Care: Key Elements Identified by Patients, Caregivers, and Healthcare Providers. A Systematic Review. Patient. 2019;12(5):461-74. Available from: https://doi.org/10.1007/s40271-019-00370-1

10. Pereira A.; Souza Da Silva R.; De Camargo C.L.; Ribeiro De Oliveira R.C. 
Volviendo a los planteamientos de la atención sensible. Enfermería Global. 2012; 25.http://revistas.um.es/eglobal/article/view/eglobal.11.1.143161

11. Castro, M.; Zeitoune, R.; Tracera, G.; Moraes, K.; Batista, K. \& Nogueira, M. Humanization in the work of nursing faculty. Revista brasileira de enfermagem. 2020;73(1):e20170855.

12. González-Hernández OJ. Validez y confiabilidad del instrumento "Percepción de comportamientos de cuidado humanizado de enfermería $3^{a}$ versión". Aquichan. 2015;15(3):381-392. DOI: 10.5294/aqui.2015.15.3.6

13. Bruce, J. Implementing the user perspective. Studies in Family Planning. 1980;11(1):29-33.

14. Diprete E. Garantía de la Calidad de la Atención de salud en los países en desarrollo. 717th ed. Bethesda; U. R. C.; 2002.

15. Eugenio Rojas, K.D.; Ortiz González, M.; Triviño Bermúdez, M. \&Velasco Peña, E.A. Percepción Del Cuidado Humanizado En Profesionales De Enfermería En Una Institución Prestado De Servicio De Salud En Urgencias. 2018; Available from: http://repository.ucc.edu.co/bitstream/ucc/7648/2/2018_Cuidado_Humanizado_Profesi onales.pdf

16. Romero-Massa, E.; Contreras-Méndez, I.; Pérez-Pájaro, Y. \& Jiménez-Zamora, V. Cuidado humanizado de enfermería en pacientes hospitalizados. Cartagena, Colombia. Revisata Ciencias Biomédicas. 2013;4(1):60-8.

17. Valdivia Cornejo, M.J. Percepción del cuidado humanizado y nivel de satisfacción en pacientes de área observación, Emergencia- Hospital Honorio Delgado. Universidad Nacional de San Agustín de Arequipa; 2019.

18. Ceballos Vásquez, P. Desde los ámbitos de enfermería, analizando el cuidado humanizado. Ciencia y Enfermeria. 2010;16(1):31-5.

19. Espinoza Medalla, L.; Huerta Barrenechea, K.; Pantoja Quiche, J.; Velásquez Carmona, W.; Cubas Cubas, D. \& Ramos Valencia, A. El cuidado humanizado y la percepción del paciente en el Hospital EsSalud Huacho. Octubre de 2010. Ciencia y Desarrollo. 2011;13:53.

20. Acosta Revollo, A.; Mendoza Acosta, C. A.; Morales Murillo, K. \& Quiñones Torres, A.M. percepción del paciente hospitalizados sobre el cuidado humanizado brindado por enfermería en una IPS de tercer nivel. Cartagena 2013. Vol. 66. Corporación Universitaria Rafael Núlez. Facultad de ciencias de la Salud; 2013.

ISSN 1695-6141

( COPYRIGHT Servicio de Publicaciones - Universidad de Murcia 